\title{
Comparison of midazolam versus clonazepam as premedication scheduled for elective abdominal hysterectomies
}

\author{
Bala Neeru', Singh Hardip ${ }^{2}$, Attri Joginder Pal ${ }^{3}$, Bansal Lipsy ${ }^{4}$, Kaur Sukhman ${ }^{5}$ \\ ${ }^{1}$ Dr Neeru Bala, MD Psychiatry, Assistant Professor, Department of Psychiatry, ${ }^{2}$ Dr Hardip Singh, MD Medicine, \\ Assistant Professor, Department of Medicine, ${ }^{3}$ Dr Joginder Pal Attri, MD Anaestheisa, Associate Professor, Department \\ of Anaesthesia, ${ }^{4}$ Dr Lipsy Bansal, Junior Resident, Department of Anaesthesia, ${ }^{5}$ Dr Sukhman Kaur, Junior Resident, \\ Department of Anaesthesia, All authors are affiliated with Government Medical College, Amritsar (Punjab), India- \\ 143001
}

Address for Correspondence: Dr Joginder Pal Attri, Email: jpattri12@yahoo.co.in

\begin{abstract}
Background and aim: Anxiety is one of the most common problem which affect the patient during surgical procedures. Thus, anxiolytics have a primary role as premedication preoperatively. With this background, we designed this study to compare anxiolytic effects of oral midazolam and oral clonazepam. Methods: Study was carried out in 80 patients scheduled for elective Abdominal hysterectomy and were divided into group of 40 each. Group M 40 patients were given Tab Midazolam $10 \mathrm{mg}, 45 \mathrm{~min}$ before surgery. Group C of 40 patients were given Tab Clonazepam $2 \mathrm{mg}$, $45 \mathrm{~min}$ before surgery with a sip of water. All hemodynamic parameters, sedation score, satisfactory score, Hamitlon Anxiety Rating Scale were noted. The patient characteristics were analysed using "Chi-square test" and inter group comparison of the parametric data was done using the "unpaired t-test using software IBM SPSS 17.0. Results: Clonazepam is better anxiolytic than midazolam and has longer duration of anxiolytic effects and with minimal side effects and better hemodynamic stability than midazolam. Conclusion: Tab Clonazepam can be used as premedication to allay anxiety in the patients undergoing elective surgeries.
\end{abstract}

Keywords: Hamilton anxiety rating scale, Clonazepam, Midazolam, Sedation, Abdominal hysterectomy

\section{Introduction}

All Patients undergoing surgery experience varying level of anxiety [1] which is dependant upon the perceived threat and outcome of the surgical procedure, pain, anesthesia and worries about the family also. Patients undergoing major surgery i.e. major abdominal, thoracic and cardiac surgery and women have higher levels of anxiety [2] eg. women undergoing invasive surgical procedures for gynaecological problems like disorders hysterectomies and other abdominal surgeries experience higher levels of state anxiety.

Various hemodynamic changes eg. tachycardia, hypertension before and during surgery are more difficult to anesthetize without complications and are more likely to experience various side effects eg. headache, vomiting and pain in the postoperative period

Manuscript received $28^{\text {th }}$ June 2016

Reviewed: $13^{\text {th }}$ July 2016

Author Corrected: $24^{\text {th }}$ July 2016

Accepted for Publication $3^{\text {rd }}$ August 2016
$[3,4]$. Therefore use of anxiolytics as premedicant for relief of anxiety is very much essential.

Also during use of RA there is pain at the puncture site, fear of needles and the recall of procedures which result in failure of RA than technical issues. It is commonly observed that the patient remains anxious, nervous and fearful during RA because of different operation room environment, noise from monitor, suction and operating equipment etc. all these factors made the use of sedation during RA [5].

Benzodiazepines are widely used as anxiolytics and sedatives prior to surgery [6]. Midazolam, a water soluble benzodiazepine is a useful agent for premedication and sedation. It has a short onset time and duration of action when compared to other benzodiazepines. In addition to anxiolysis, it also 
possesses hypnotic, anticonvulsant, muscle relaxant and antegrade amnestic properties $[7,8]$.

Another benzodiazepine i.e. clonazepam may also be used as a premedicant drug to relieve anxiety preoperatively. Clonazepam is also a long acting benzodiazepine which is primarily used to control seizure attacks. It has also been prescribed for cases of panic disorder. However, there is still little information on the efficacy of Clonazepam for anxiety control in patients undergoing surgery $[9,10,11]$. Therefore, this study is an attempt to the anxiolytic effect of clonazepam before surgery.

\section{Material and Methods}

After approval from institutional Ethics Committee and obtaining informed consent, a double blinded, randomized controlled trial was conducted on 80 ASA I and II patients, aged between 20 and 60 years females, scheduled for elective abdominal hysterectomy. Patients with history of psychiatric illness, alcohol abuse, hypertension, ischemic heart disease, diabetes mellitus, patients receiving $\beta$-blockers and $\mathrm{Ca}++$ channel blockers and those taking benzodiazepines since admission to hospital were excluded from the study. Patients were equally divided into two groups with patients in group $\mathrm{M}$ receiving tab midazolam $10 \mathrm{mg}$ and group $\mathrm{C}$ receiving tab clonazepam $2 \mathrm{mg} 45$ minutes before surgery with a sip of water. Doses were calculated using equipotent doses chart. Drug was given to the patient in the pre operative area by a person not involved in taking observations. Our primary aim was to evaluation of sedation and anxiolysis with midazolam, clonazepam as a premedication for abdominal hysterectomies under regional anesthesia and to compare the satisfactory level of patients anxiety using Hamilton anxiety rating scale. Our secondary aim was to evaluate any adverse cardiovascular and respiratory events arising out of using these drugs.

Statistical analysis: The data from the present study was systematically collected, compiled and statistically analyzed using software IBM SPSS 17.0 (IBM Chicago SPSS Inc) to draw relevant conclusions. Data was expressed as mean and standard deviation, number and percentages. The patient characteristics were analyzed using the "Chi-square tests" and the inter group comparison of the parametric data was done using the "unpaired $t$-test. The $P$ value was finally determined to evaluate the level of significance. $P<0.05$ was considered as significant at $5 \%$ significance level; $P<$ 0.01 was considered significant at $1 \%$ significance level and $P<0.001$ was considered highly significant.

\section{Results}

In the present study, both groups were comparable with respect to demographic characteristics, hemodynamic parameters and side effects at various time intervals. As demographic variables, hemodynamic parameters, sedation scoring, Hamilton anxiety scoring $[12,13]$ shown in table $1,2,3,4$ respectively.

Table-1: Demographic variables.

\begin{tabular}{|c|c|c|c|}
\hline & Group C & Group M & p value \\
\hline AGE & $42.67 \pm 5.64$ & $44.24 \pm 6.33$ & NS \\
\hline WEIGHT & $63.69 \pm 9.39$ & $60.37 \pm 16.26$ & NS \\
\hline HEIGHT & $153.57 \pm 11.27$ & $156.22 \pm 14.06$ & NS \\
\hline ASA GRADE & 23 & & \\
I & 17 & 15 & \\
II & 25 & \\
\hline
\end{tabular}

Table -2: hemodynamic parameters.

\begin{tabular}{|c|c|c|c|}
\hline & Group C & Group M & p value \\
\hline SBP Before drug & $127.31 \pm 11.97$ & $125.31 \pm 11.97$ & NS \\
SBP After drug & $112.83 \pm 11.39$ & $110.83 \pm 11.39$ & NS \\
\hline DBP Before drug & $82.52 \pm 8.77$ & $83.52 \pm 8.77$ & NS \\
DBP After drug & $72.13 \pm 6.74$ & $73.13 \pm 6.74$ & \\
\hline HR Before drug & $80.87 \pm 8.29$ & $84.87 \pm 8.29$ & $86.32 \pm 6.58$ \\
HR After drug & $82.32 \pm 6.58$ & & \\
\hline
\end{tabular}


Table- 3: Sedation score.

\begin{tabular}{|c|c|c|c|}
\hline & Group C & Group M & p value \\
\hline Mean of sedation score & $1.85 \pm 0.56$ & $1.77 \pm 0.56$ & NS \\
\hline Duration of sedation & $11.45 \pm 0.72 \mathrm{hrs}$ & $.45 \pm .15 \mathrm{hrs}$ & $<0.001 \mathrm{HS}$ \\
\hline
\end{tabular}

Table-4: Hamilton anxiety scoring.

\begin{tabular}{|c|c|c|c|}
\hline Time & Group C (HAM-A) & Group M (HAM-A) & p value \\
\hline $0 \mathrm{~min}$ & 27 & 26 & NS \\
\hline $45 \mathrm{~min}$ & 18 & 21 & $<0.01, \mathrm{~S}$ \\
\hline $90 \mathrm{~min}$ & 19 & 26 & $<0.001, \mathrm{HS}$ \\
\hline $120 \mathrm{~min}$ & 19 & 26 & $<0.001, \mathrm{HS}$ \\
\hline
\end{tabular}

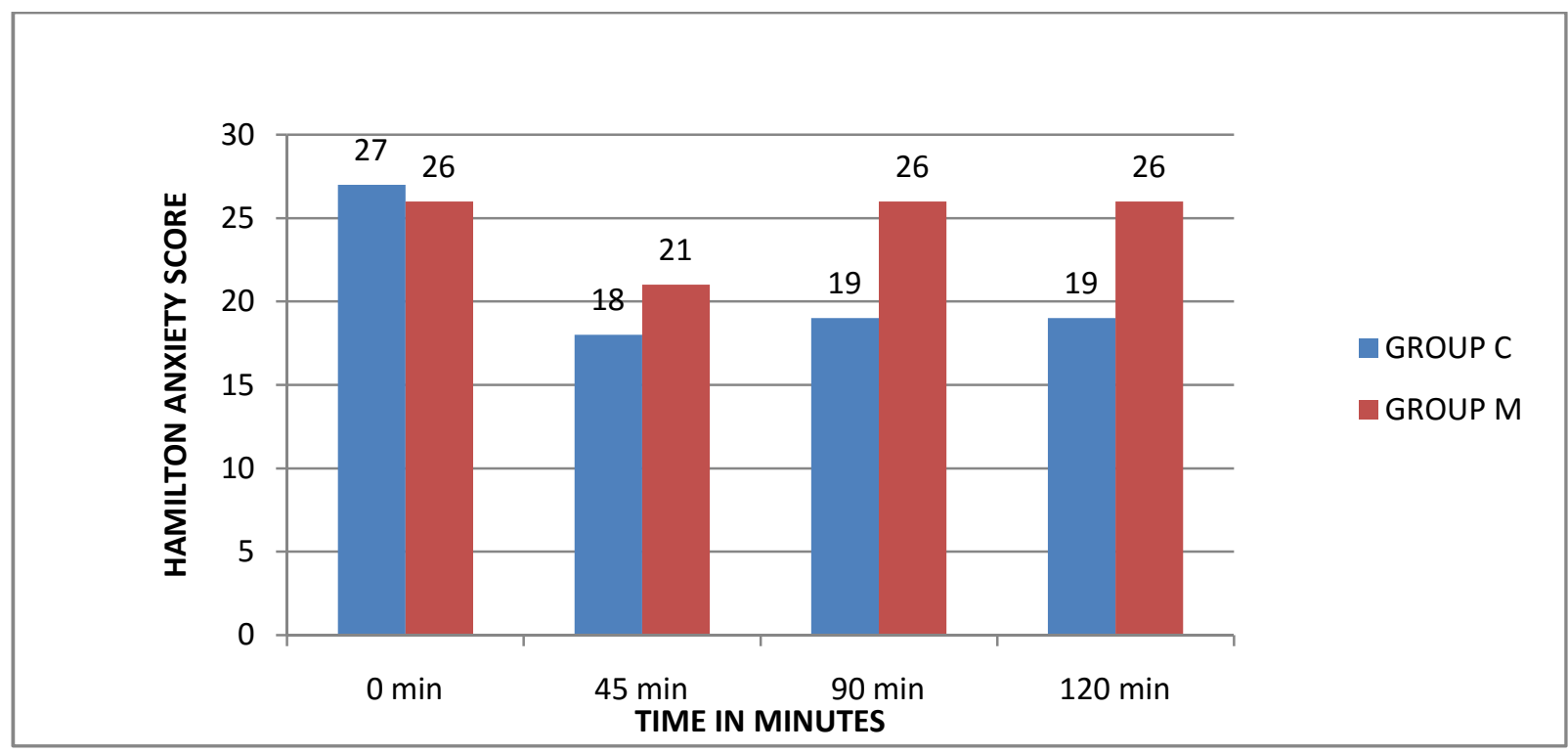

Graph 1: Showing Hamilton anxiety scoring

Haemodynamic parameters including systolic blood pressure, diastolic blood pressure and heart rate as objective indicators of anxiolysis. There was a statistically significant difference in both the systolic and diastolic blood pressures ( $\mathrm{p}$ value $>0.05$ ). As for as heart rate is concerned, it reduction in both the systolic and diastolic blood pressures whereas the heart rate increased significantly clinically but not there was no any statistically significance between two groups.t, in both groups from baseline but there was no statistically significant ( $p$ value $<0.05$ ) difference between two groups.

Sedation score was compared in post operative period. Patients of Group C have longer hours of sedation and remained sedated for about 10-12 hours after surgery which was helpful in patients of abdominal hysterectomy as patients were admitted in ward for post operative care for 1-2 days.

Hamilton Anxiety Rating Scale (HAM) scoring done at 0 minutes, 45 minutes, 60 minutes, 90 minutes and 120 minutes. There was a significant difference in HAM scale in 2 groups. But there was statistical differences of level of anxiety in Groups $\mathrm{M}$ and $\mathrm{C}$ at four different times of study. In Groups M and C, total mean HAM score was 26 and 27 respectively showing moderate to severe anxiety which was observed at 0 minute before giving pre medication. After 45 minutes, the mean HAM score was 18 and 21 in Group C and M respectively with statistical significant difference of p value. 90 minutes after giving pre medication HAM in Group C was 19 and in Group M was 26 with statistical significant difference $(p<0.001)$. There was no statistically significant difference between two groups at baseline values ( $p$ value $>0.05$ ) at baseline but when the two groups were compared after 45 minutes intervals, there was significant statistical difference (as compared to at $45 \mathrm{~min}$ after premedication $\mathrm{p}$ value $<0.001$ ) which was statistically highly significant. 


\section{Discussion}

Pre-medication has been used to achieve several purposes including reduction of anxiety, preamptive analgesia, antisialogogue effect, provision of sedation etc but the primary purpose of prescribing these drugs in the immediate preoperative period is to allay patient's anxiety. During RA patient had also pain at the puncture site, fear of needles and the recall of procedures in these cases Sedation increases comfort level of the patient and acceptance of RA and decreases the analgesic requirement [5]. Benzodiazepines are the most commonly used drugs for this purpose. Midazolam is the most commonly used anxiolytic drug. Other benzodiazepines that can be used for this purpose are diazepam, lorazepam, clonazepam etc. We performed a double blind, randomized controlled study to assess the anxiolytic effect of midazolam and clonazepam as anxiolyttic subjectively, objectively and biochemically using HAM anxiety scores and sedation scores.

Midazolam is a benzodiazepine that acts by enhancing the effect of the neurotransmitter GABA at the GABAA receptor resulting in sedative, hypnotic, anxiolytic, anti convulsant and muscle relaxant properties. These properties make midazolam useful in treating anxiety, seizures, muscle spasam and as pre medication for various procedures $[7,8]$.

Clonazepam is also a benzodiazepine drug which acts by binding to benzodiazepine site of the GABA receptor enhancing the effect of GABA binding on the neurons. As Clonazepam is effective in low doses it is set to be among the class of highly potent benzodiazepine. Clonazepam is used as a first line treatment for acute seizures and has been found effective in treating anxiety disorder and panic disorder $[9,10]$.

The dose of Midazolam $10 \mathrm{mg}$ and Clonazepam $2 \mathrm{mg}$ were used as these are the highest, safe single doses used in previous studies. The drug was given 45 minutes before surgery with a sip of water under direct observation. Their administration 45 minutes prior to surgery appeared rationale in order to attain maximal plasma concentration at the time of surgical stimuli.

Though Midazolam is rapidly absorbed (peak within 20 minutes) and Clonazepam (peak within 30-40 minutes), sufficient time is given to both the drugs to achieve peak effect. In our study in both the groups sedation score was better after premedication but there was no statistical significant difference between two groups. Similar results were found by Khan EI et al and Zamiri E et al $[8,10]$.

We also measured the hemodynamic parameters including systolic blood pressure, diastolic blood pressure and heart rate as objective indicators of anxiolysis. In this study, there was a statistically significant reduction in both the systolic and diastolic blood pressures whereas the heart rate increased clinically but not statistically significant, in both groups from baseline but there was no statistically significant difference between two groups. The results of our study are consistent with previous studies $[8,10]$.

We observed that the preoperative single dose of clonazepam $2 \mathrm{mg}$ is a better pre medication than $10 \mathrm{mg}$ of Midazolam in relieving anxiety and better sedation score with longer duration of action which is beneficial especially in patients staying in hospital postoperatively for 1-2 days.

As in our study patients undergoing elective abdominal hysterectomies under spinal anesthesia, have significantly lower HAM scores and longer duration of action in Group C as compared to Group M. So it is concluded from our study that midazolam and clonazepam significantly reduce the patient's anxiety without altering the intraoperative hemodynamics. But Clonazepam has been found to be better anxiolytic than Midazolam.

\section{Conclusion}

Clonazepam is better anxiolytic, hemodynamically stable with longer duration of action than midazolam. Thus, it can replace midazolam as oral premedication in patients scheduled.

Funding: Nil, Conflict of interest: None initiated, Permission from IRB: Yes

\section{Bibliography}

1. Scott LE, Clum GA, Peoples JB. Pre-operative predictors of postoperative pain. Pain. 1983 Mar;15 (3):283-93.

2. Mitchell MJ. Psychological preparation for patients undergoing day surgery. Ambul Surg 2000;8:19-29. 
3. Mitchell M. Patients' perceptions of pre-operative preparation for day surgery. J Adv Nurs. 1997 Aug; 26(2):356-63.

4. Carr E1, Brockbank K, Allen S, Strike P.. Patterns and frequency of anxiety in women undergoing gynecological surgery. J Clin Nurs. 2006 Mar; 15(3):341-52.

5. Attri JP, Gupta KK, Khetarpal R. Emerging trends of sedation during regional anesthesia. Anaesth Pain \& Intensive Care 2015; 19(4):527-532.

6. White PF. Pharmacologic and clinical aspects of preoperative medication. Anesth Analg. 1986 Sep; 65 (9):963-74.

7. Reves JG, Fragen RJ, Vinik HR, Greenblatt DJ. Midazolam: pharmacology and uses. Anesthesiology 1985 Mar;62(3):310-24.

8. Khan EI, Kamal RS, Ullah H. Anxiolytic effect of midazolam premedication assessed by clinical and platelet aggregation profiles. J Ayub Med Coll Abbottabad 2010 Apr-Jun;22(2):4-7.

9. Stahl Y, Persson A, Petters I, Rane A, Theorell K, Walson P. Kinetics of clonazepam in relation to electroencephalographic and clinical effects. Epilepsia 1983 Apr;24(2):225-31.

10. Zamiri B, Eftekharian HR, Arasteh N. Clonazepam for the Management of Anxiety Associated with Oral Surgery: A Randomized Double-blind Controlled Trial. J Dent Shiraz Univ Med Scien. 2012 June; 13(2): 75-79

11. Cloos JM. The treatment of panic disorder. Curr Opin Psychiatry. 2005 Jan;18(1):45-50.

12. Hamilton M.The assessment of anxiety states by rating. Br J Med Psychol 1959; 32:50-55.

13. Maier W, Buller R, Philipp M, Heuser I. The Hamilton Anxiety Scale: reliability, validity and sensitivity to change in anxiety and depressive disorders. J Affect Disord 1988;14(1):61-8

\section{How to cite this article?}

Bala Neeru, Singh Hardip, Attri Joginder Pal, Bansal Lipsy, Kaur Sukhman. Comparison of midazolam versus clonazepam as premedication scheduled for elective abdominal hysterectomies. Int J Med Res Rev 2016;4(8):13301334.doi:10.17511/ijmrr.2016.i08.09. 